\title{
A Mobility Model for the Realistic Simulation of Social Context
}

\author{
Daniel Fischer \\ SAP AG, Germany \\ daniel.fischer@sap.com
}

\begin{abstract}
Simulation is a fundamental means for evaluating mobile applications based on ad-hoc networks. In recent years, the new breed of social mobility models (SMMs) has risen. Contrary to most classical mobility models, SMMs model the social aspects of human mobility, i.e. which users meet, when and how often. Such information is indispensable for the simulation of a wide range of socially-aware communication protocols mostly based on delay-tolerant networks, including opportunistic ad-hoc routing and data dissemination systems. Each SMM needs a model of the relations between a set of relevant people (called social network model - SNM) in order to simulate their mobility. Existing SMMs lack flexibility since each of them is implicitly restricted to a specific, simplifying SNM.

We present GeSoMo (General Social Mobility Model), a new SMM that separates the core mobility model from the structural description of the social network underlying the simulation. This simple and elegant design principle gives GeSoMo generalizing power: Arbitrary existing and future SNMs can be used without changing GeSoMo itself. Our evaluation results show that GeSoMo produces simulations that are coherent with a broad range of empirical data describing real-world human social behavior and mobility.
\end{abstract}

Digital Object Identifier 10.4230/OASIcs.KiVS.2011.215

\section{Introduction}

In recent years, powerful mobile devices with short-range wireless network interfaces have become widely used that leverage the idea of mobile ad-hoc networks. Connectivity between mobile nodes is created sporadically and highly depends on the movement of the users carrying those devices. This human movement is strongly influenced by social relations $[13,16]$ : We do not meet other people on a purely random basis; instead, we meet some people more frequently and regularly than others, e.g. friends, colleagues, family. We call the network of social relationships of a larger group of people a social network.

Since the social network directly influences the mobility of people (and therefore those peoples' devices), many protocols and distributed algorithms, mostly in the area of opportunistic networks, exploit the structural properties of this underlying social network $[2,5,15,16]$. To evaluate such approaches, several social mobility models (SMMs) have been proposed recently $[3,8,11,17]$. The general task of a SMM is to simulate the encounters between mobile users such that they meet according to their social relationships. A SMM is always (possibly implicitly) based on a social network model (SNM) defining the structure of the relations between mobile users. The existing SMMs, however, have drawbacks. Each of them is tightly integrated with a specific SNM. Thus, by selecting an SMM, the SNM is fixed and cannot be changed. This is disadvantageous since there are a number of competing SNMs, and the selection of the right SNM is scenario-dependent and can be subject to debate. Furthermore, the used SNMs are quite simplistic which can have significant consequences on the evaluation results of applications simulated on top of it. SNMs with more realistic properties (e.g. [19]) are not used by existing SMMs. Also note that social network research is still a young discipline such that the SNMs are not yet established and improved models will be 
proposed in the future. Finally, none of the existing SMMs models group mobility, i.e. the fact that humans tend to move in groups because of their social relations.

This paper provides an overview of our work in [6]. We introduce GeSoMo (General Social Mobility Model) [7], a new SMM that generalizes a number of existing models by separating the social mobility model and the social network model on which the SMM is based. GeSoMo receives a social network as input and creates a mobility pattern simulating a series of encounters between the users in this network. Thus, arbitrarily structured social networks can be used without changing the simulation model. Additionally, a number of parameters may be used for tuning GeSoMo to different scenarios. We show that GeSoMo produces realistic mobility patterns by comparing its characteristics to real measurements of human mobility.

\section{Spatio-temporal Characteristics of Human Mobility}

Apart from the fact that human mobility is influenced by the underlying social network, the investigation of real human mobility traces has shown several omnipresent spatio-temporal characteristics that a realistic SMM should capture:

- Inter-contact times, i.e. the time intervals between two successive contacts (connectivity) of two nodes, are of particular interest since they strongly influence the speed of relaying information in the social network and, thus, in any communication network resulting from it. Independent of the investigated trace, the inter-contact distribution is characterized by a dichotomy: There is a power-law up to an inter-contact time of about half a day, thereafter followed by an exponential decay $[4,14]$.

- The probability that individuals return to previously visited locations after a certain time $t$ is characterized by distinct peaks at multiples of a day [9]. This temporal regularity confirms the intuition behind human habitual behavior.

- Spatial Regularity: Individuals have a strong preference for a small number of locations while visiting all other locations only with low probability [9].

- Frequently, humans do not move independently of each other from location to location, but rather move as a group. Group mobility is a realistic human characteristic that is already exploited by existing mobile applications [18], e.g. by identifying groups and using their combined resources.

\section{The General Social Mobility Model}

In this section, we first introduce our idea of how the simulated mobility should conform to the underlying social network. Subsequently, we give a brief overview of the concepts to realize a mobility model which both exhibits a high conformance and realistic spatio-temporal characteristics.

\subsection{Conformance}

The general idea behind GeSoMo is show in Figure 1. It takes an arbitrary social network as input. A social network is essentially a graph where nodes represent people and weighted edges represent social relationships. The weight is an indicator of the strength of the social relation. Two people having a social relation are called social acquaintances. Social networks can be generated by a number of social network models (SNMs), e.g. $[10,19,20]$ describing different typical structural properties (e.g. scale-free node degree, high clustering, ...). GeSoMo then translates the static social network into realistic spatio-temporal mobility characteristics described by a mobility trace. This trace can be used as input to a simulation tool such as ns2 [1]. Such simulation tools determine at which time two nodes have connectivity resulting in a connectivity graph. At the moment, we 


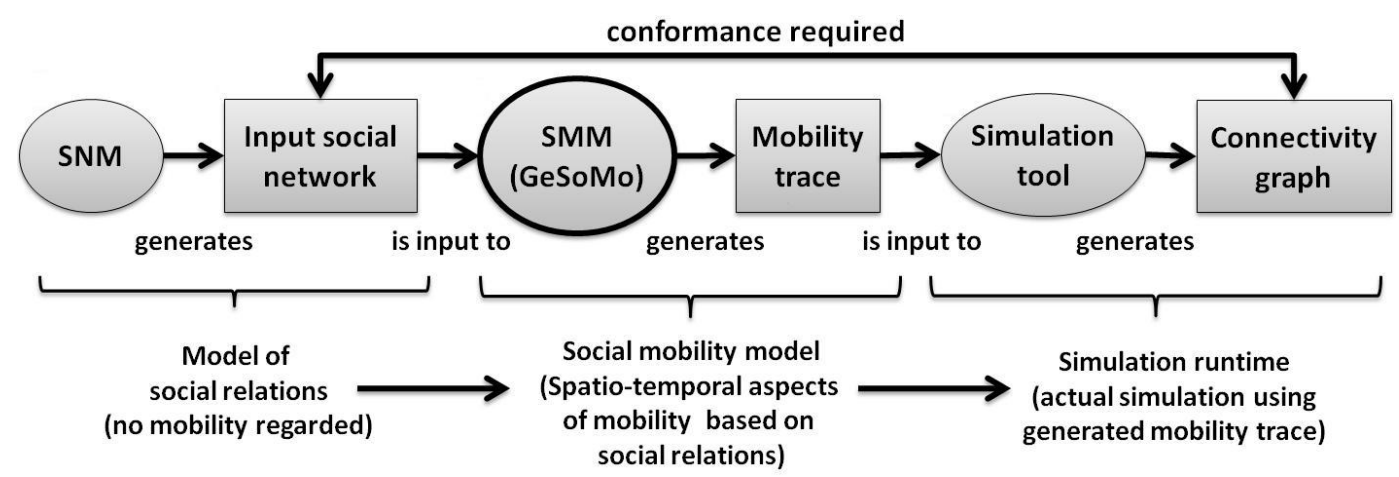

Figure 1 Dependencies between social network and simulated mobility

know that important structural properties of the input social network are mapped to the connectivity graph $[13,16]$ and the frequency and duration of contacts between social acquaintances is typically an order of magnitude larger than unrelated node pairs [16]. However, so far, social studies do not show how exactly the strength of social relations relate to the number of contacts (i.e. times of connectivity) between nodes. Hence, we define conformance as follows: The number of contacts between nodes should be proportional to the strength of the relationship. Note, however, that this interpretation can be adjusted in an arbitrary way, without changing GeSoMo, as soon as corresponding future results in social studies are known (for details, see [6]).

\subsection{Outline of the General Concepts}

For each node in the input social network, GeSoMo simulates a mobile node (or short: node) assuming that the corresponding user carries a single mobile device. Social interactions take place at so-called anchors which are an abstract representation of real locations (e.g. a bus stop or a coffee shop). Nodes move between anchors, and if a node reaches an anchor, it stays there for a specific duration, before it starts to move towards the next anchor. The movement between anchors can be performed by several nodes together in order to simulate group mobility. GeSoMo allows to configure the frequency of such group movements.

The probability that a node chooses a certain anchor as its next desination is proportional to its anchor attraction which consists of three additive components: First, anchors themselves exert a time-varying attraction on nodes: Nodes are attracted to a subset of anchors leading to a spatial regularity and this attraction is periodic which creates a temporal regularity. Second, nodes are also attracted by other nodes currently traveling to or residing at certain anchors. This node-based attraction depends on the strengths of the social relations in order to yield frequent meetings between social acquaintances. Third, in cases where nodes do not have a social relation with each other, it is necessary that nodes are repulsed (i.e. negatively attracted) by each other. Both node-based types of attractions (positive and negative) are necessary in order to yield a high conformance.

Influences such as different travel-times between anchors may lead to less or more meetings between some pairs of social acquaintances than required by the conformance criterion. To achieve strong conformance independent of such disturbances, we propose a feedback control mechanism: If too many meetings between two nodes have been produced, their social attraction is decreased. Conversely, if too few meetings have been produced, their social attraction is increased. Hence, after the adjustment, less (or more) meetings between them will occur, such that GeSoMo yields a robust conformance despite physical effects such as heterogeneous speed/anchor distributions, obstacles, or streets. 


\begin{tabular}{|c|c|c|c|}
\hline SNM & Caveman & Toivonen & Holme-Kim \\
\hline Percentage Matched & $99.91 \%$ & $99.89 \%$ & $99.90 \%$ \\
\hline 95\% Conf. Int. & $0.15 \%$ & $0.11 \%$ & $0.11 \%$ \\
\hline
\end{tabular}

Table 1 Results of the conformance metric (standard parameters)

\section{$4 \quad$ Evaluation Results}

In this section, we show that GeSoMo conforms to arbitrary structural properties of the input social network with high accuracy and still produces realistic spatio-temporal properties. We show the latter by evaluating several known omnipresent characteristics of human behavior (cf. Section 2) and comparing them to results of real measurements. We used three different SNMs (Caveman model [20], Holme-Kim model [10], Toivonen model [19]), for generating input social networks to show that the results detailed in this section are independent of the concrete SNM (and hence, their structural properties). The detailed simulation setup can be found in [6].

\subsection{Conformance}

We proposed the following metric to measure the conformance to the input social network: Assume a node $u$ has a social relation with node $v$ and node $w$, and the strength of the relation with $v$ is signficantly stronger than the relation with $w$. Then, based on the conformance criterion, we expect that $u$ has more contacts with $v$ than with $w$ after a simulation runtime of sufficient length. The percentage of node pairs for which this was true is shown in Table 1 for different input SNMs.

The results clearly show that the conformance is very high independent of the SNM used to generate input social networks. Furthermore, we have performed simulations with a broad range of parameters (see $[6,7]$ ) and report that the conformance exceeds $99 \%$ in all cases with each of the three used SNMs. This shows that GeSoMo is characterized by a high conformance independent of the concrete parameters of our model. Among others, this good result is mainly due to the proposed concept of robust conformance.

\subsection{Inter-contact Characteristics}

In the following, we compare the inter-contact distribution of GeSoMo to the Infocom-trace [12]. This mobility trace is one of the few available direct-contact traces that has a high number of representative contacts. At the same time, its power-law/exponential decay dichotomy is representative for many other traces. Figure 2 shows the complementary aggregated inter-contact CDF of GeSoMo for three different SNMs in comparison to the Infocom-trace. Independent of the used SNM, we observe a distinct power-law (straight line in a log-log plot) followed by an exponential degradation after about half a day $(\approx 40000 s)$ in all cases. These results correspond to the omnipresent dichotomy found in real human mobility traces $[4,14]$. GeSoMo exhibits this characteristic for a broad range of parameters $[6,7]$.

\subsection{Spatial and Temporal Regularity}

In order to investigate the characteristics of spatial regularity, Gonzales et al. have analyzed the reappearance of humans at specific locations in the trajectories of 100000 mobile phone users [9]. For each location, they ranked the number of visits where rank $L$ represents the $L$-most visited location. They report that the probability of finding a user at a location of rank $L$ is well approximated by $P(L) \sim L^{-1}$ independent of the total number of locations a user has visited. We have performed a similar measurement for GeSoMo in order to conduct a comparison. Figure 3 shows the average 


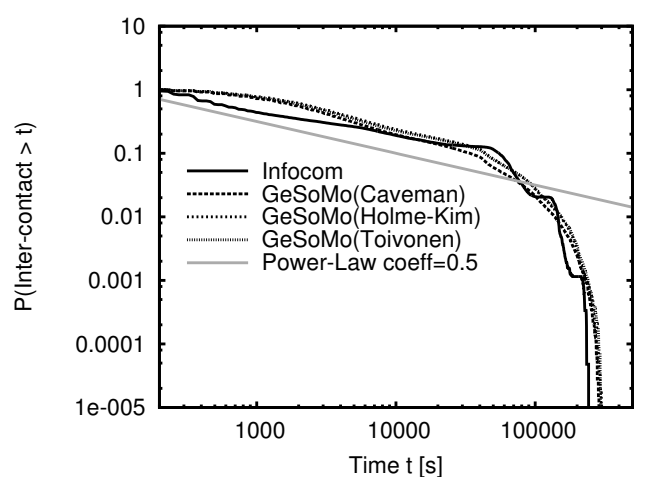

Figure 2 Inter-contact distribution in comparison to the Infocom-trace

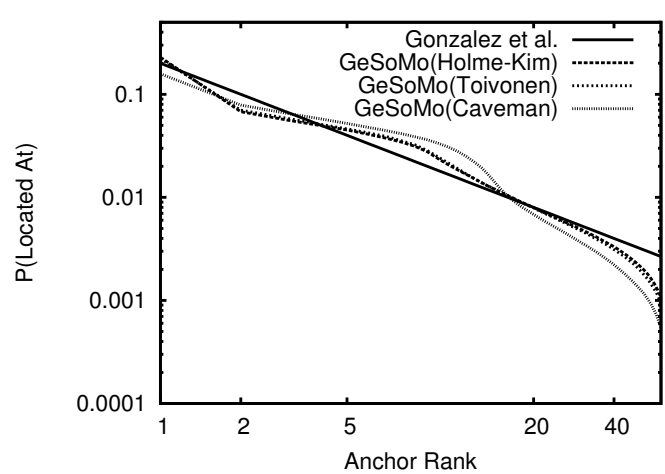

Figure 3 Spatial characteristics compared to an approximation of real-world mobility

visiting probability over all nodes for each anchor rank in comparison to the approximation of Gonzales et al. The good fit shows that nodes in GeSoMo have a preference for a small subset of anchors which they visit with high probability, very similar to real-world mobility behavior.

The evaluation GeSoMo's temporal regularty shows the tendency of users to periodically return to certain locations with high probability (see [6] for details). Very similar characteristics were also reported for existing real traces of human mobility.

\section{Conclusions and Outlook}

We have presented GeSoMo, a new social mobility model that can be used to evaluate applications for people-based delay tolerant and opportunistic networks. GeSoMo generalizes a number of existing social mobility models by leveraging the elegant and simple concept of separating the simulation model from the structural description of the simulated social network. GeSoMo builds on the principle of conformance: an arbitrary input social network is simulated such that the connectivity graph produced among the simulated mobile devices conforms to this input network as closely as possible. This enables researchers to use any existing social network model that is appropriate to their scenario and also the (more realistic) ones that will be discovered in the future without changing the mobility model. This is a major step forward compared to the existing social mobility models which build on specific simplifying social network models and, thus, limit the flexibility of a simulation severely.

In our evaluation which is based on a broad range of empirical observations and data of real user behavior, we have shown that GeSoMo creates simulations that behave realistically with respect to 1 . inter-contact distribution, 2. spatial characteristics, and 3. temporal characteristics of human behavior. Furthermore, we have shown that the conformance is very high for all used SNMs. Hence, it is the first SMM which shows all these omnipresent characteristics of human mobility. It is also the first SMM that incorporates group mobility. All these results are independent of the model parameters. We conclude that GeSoMo is a major step forward in the field of social mobility models due to its flexibility and generalizing power over the used SNM.

\section{References}

1 The network simulator - ns-2. http://www.isi.edu/nsnam/ns/.

2 Jó Ágila Bitsch Link, Nicolai Viol, André Goliath, and Klaus Wehrle. SimBetAge: Utilizing Temporal Changes in Social Networks for Delay/Disconnection Tolerant Networking. In MobiQuitous 2009, 2009. 
3 Chiara Boldrini, Marco Conti, and Andrea Passarella. Users mobility models for opportunistic networks: the role of physical locations. In IEEE Wireless Rural and Emergency Communications, 2007.

4 Augustin Chaintreau, Pan Hui, Christophe Diot, Richard Gass, and James Scott. Impact of human mobility on opportunistic forwarding algorithms. IEEE Transactions on Mobile Computing, 6(6):606-620, 2007.

5 Elizabeth M. Daly and Mads Haahr. Social network analysis for routing in disconnected delaytolerant manets. In MobiHoc '07: Proceedings of the 8th ACM international symposium on Mobile ad hoc networking and computing, pages 32-40, 2007.

6 Daniel Fischer. A mobility model for the realistic simulation of social context. Master's thesis, University of Stuttgart, 2009.

7 Daniel Fischer, Klaus Herrmann, and Kurt Rothermel. GeSoMo - A General Social Mobility Model for Delay Tolerant Networks. In Proceedings of the 7th IEEE International Conference on Mobile Ad-hoc and Sensor Systems (MASS '10), pages 1-9. IEEE, 2010.

8 Sabrina Gaito, Giuliano Grossi, and Federico Pedersini. A two-level social mobility model for trace generation. In MobiHoc '08: Proceedings of the 9th ACM international symposium on Mobile ad hoc networking and computing, pages 457-458, 2008.

9 M. C. Gonzalez, C. A. Hidalgo, and A. L. Barabasi. Understanding individual human mobility patterns. Nature, 453:779-782, 2008.

10 Petter Holme and Beom Jun Kim. Growing scale-free networks with tunable clustering. Phys. Rev. E, 65(2):026107-026112, 2002.

11 W. Hsu, T. Spyropoulos, K. Psounis, and A. Helmy. Modeling Time-variant User Mobility in Wireless Mobile Networks. In Proceedings of IEEE INFOCOM, 2007.

12 P. Hui, A. Chaintreau, J. Scott, R. Gass, J. Crowcroft, and C. Diot. Pocket switched networks and human mobility in conference environments. In Proceedings of the 2005 ACM SIGCOMM workshop on Delay-tolerant networking, pages 244-251, 2005.

13 Pan Hui. People are the network: experimental design and evaluation of social-based forwarding algorithms. Technical report, UCAM-CL-TR-713, University of Cambridge, 2007.

14 Thomas Karagiannis, Jean-Yves Le Boudec, and Milan Vojnović. Power law and exponential decay of inter contact times between mobile devices. In Proceedings of the 13th ACM conference on Mobile computing and networking, pages 183-194, 2007.

15 A.G. Miklas, K.K. Gollu, K.K.W. Chan, S. Saroiu, K.P. Gummadi, and E. De Lara. Exploiting social interactions in mobile systems. Lecture Notes in Computer Science, 4717:409-428, 2007.

16 A. Mtibaa, A. Chaintreau, J. LeBrun, E. Oliver, A.K. Pietilainen, and C. Diot. Are you moved by your social network application? In Proceedings of the first workshop on Online social networks, pages 67-72, 2008.

17 Mirco Musolesi and Cecilia Mascolo. A Community based Mobility Model for Ad Hoc Network Research. In Proceedings of the 2nd ACM/SIGMOBILE International Workshop on Multi-hop Ad Hoc Networks: from theory to reality, 2006.

18 M. Thomas, A. Gupta, and S. Keshav. Group based routing in disconnected ad hoc networks. Lecture Notes in Computer Science, 4297:399-410, 2006.

19 R. Toivonen, J.P. Onnela, J. Saramäki, J. Hyvönen, and K. Kaski. A model for social networks. Physica A: Statistical Mechanics and its Applications, 371(2):851-860, 2006.

20 D.J. Watts. Small worlds: the dynamics of networks between order and randomness. Princeton University Press, 1999. 